\title{
Studying frequency correlations of photon pairs generated via spontaneous four-wave mixing in a microring resonator
}

\author{
Ilya Chuprina ${ }^{1,2^{*}}$, and Alexey Kalachev ${ }^{1,2}$ \\ ${ }^{1}$ Kazan E. K. Zavoisky Physical-Technical Institute, 10/7 Sibirsky tract Str., Kazan, 420029 Russia \\ ${ }^{2}$ Kazan Federal University, 420008 Kazan, Russia
}

\begin{abstract}
Spontaneous four-wave mixing (SFWM) in a microring resonator is considered theoretically. The joint spectral amplitude and Schmidt number of generated biphoton field are calculated numerically for different pump pulses. Optimal pulse shapes that provide maximum efficiency of SFWM and minimum frequency correlations between signal and idler photons are found.
\end{abstract}

The nonlinear process of spontaneous four-wave mixing (SFWM) is a promising way to build heralded single-photon and entangled two-photon sources, which are essential elements in a number of photonic quantum technologies [1]. Integration of such sources on-chip is considered as the most promising approach to making them compatible with existing photonic architectures and scaling the optical schemes [2]. In doing so, to reduce the required pump power from the milliwatt to the microwatt regime, microring resonators prove to be very useful [3], wherein the rate of SFWM increases as the mode volume decreases, and the larger free-spectral range makes it easier to extract the emitted photons. It has been shown recently that short and broadband pump pulses allow reducing or even eliminating frequency correlation between the emitted photons $[4,5]$, which is crucial for preparing pure heralded single-photon states. In the present work, we study the dependence of frequency correlation and efficiency of photon pair generation on the pump pulse waveform.

The SFWM process involves sending a pump laser beam into a third-order nonlinear material so that the nonlinear interaction occasionally leads to the annihilation of two pump photons and creation of two photons in other modes (referred to as signal and idler) under conditions of energy conservation and phase matching. The quantum mechanical two-photon state is described by the joint spectral amplitude (JSA) $F\left(\omega_{s}, \omega_{i}\right)$, which is the probability amplitude of measuring a pair of photons at frequencies $\omega_{s}$ and $\omega_{i}$. In possession of JSA we can calculate the Schmidt number $\mathrm{K}$, which describes frequency correlation of the twophoton state. In the present work, we consider SFWM in a silicon-nitride racetrack resonator on silicon dioxide substrate and follow the theory developed in [6]. The joint spectral amplitude of biphoton field is calculated numerically for different pump pulses such as Gaussian, hyperbolic secant, bilateral exponential and rising exponential pulse. In doing so, we consider different values of ratio between the pump resonance linewidth $\delta \omega_{p}$ and

* Corresponding author: ilya.chuprina@gmail.com 
signal/idler resonance linewidth $\delta \omega_{s, i}$. Some results of the calculation are shown in Fig. 1, where the joint spectral intensity (JSI) $\left|F\left(\omega_{s}, \omega_{i}\right)\right|^{2}$ and the corresponding Schmidt number are displayed for Gaussian and rising exponential pulses.
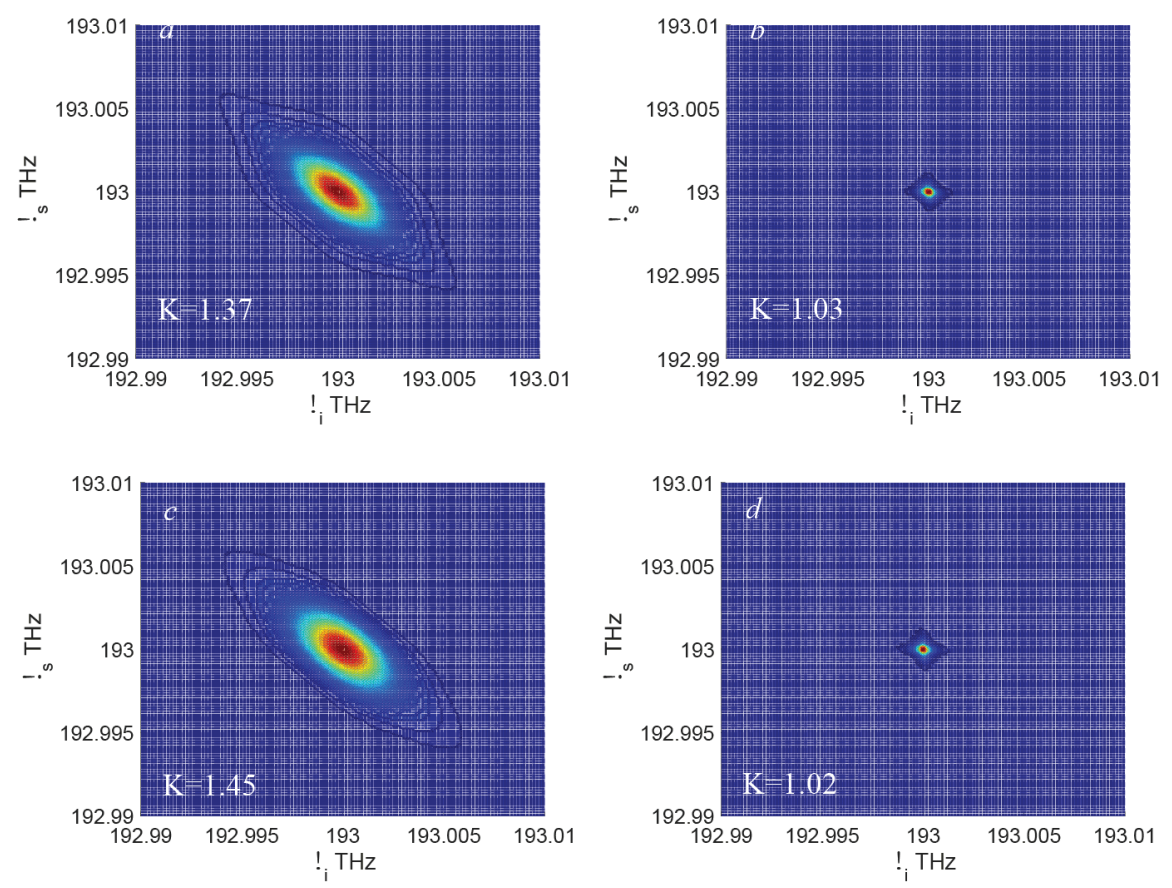

Fig. 1. The calculated JSI and Schmidt number of the biphoton field for rising exponent pump pulse $(a),(b)$ and for Gaussian pump pulse (c), (d), and for different pump resonance linewidth: $\delta \omega_{p}=\delta \omega_{s, i}$ (a), (c) and $\delta \omega_{p}=10 \delta \omega_{s, i}(b),(d)$. In all cases, the temporal duration of the pump pulses is optimized to provide maximum efficiency.

According to the obtained results, among considered pulse shapes, Gaussian pulses provide maximum efficiency for any value of $\delta \omega_{s, i} / \delta \omega_{p}$. When $\delta \omega_{p}=\delta \omega_{s, i}$, the rising exponential pulse, which is the optimal for excitation of a single cavity mode, is less effective but provides the minimum Schmidt number. However, in the case of $\delta \omega_{p} \gg \delta \omega_{s, i}$, which is necessary for generating factorable two-photon states $[4,5]$, minimum Schmidt number is provided by bilateral exponential pulses.

This work was supported by the Russian Science Foundation (project No. 16-12-00045).

\section{References}

[1] J.L. O’ Brien, A. Furusawa, J. Vuckovic, Nature Photonics 3, 687 (2009)

[2] S. Tanzili, A. Martin, F. Kaiser et al., Las. Photon. Rev. 6, 115143 (2012)

[3] W. Bogaerts, P. De Heyn, T. Van Vaerenbergh et al., Las. Photon. Rev. 6, 47 (2011)

[4] L.G. Helt, Z. Yang, M. Liscidini, J.E. Sipe, Opt. Lett. 35, 3006 (2010)

[5] Z. Vernon, M. Menotti, C.C. Tison et al., Opt. Lett. 42, 3638 (2017)

[6] I.N. Chuprina, P.P. An, E.G. Zubkova et al. Quant. Electron. 47(10), 887 (2017) 